\title{
La construcción política de la frontera. Entre los discursos nacionalistas y la "producción” de trabajadores precarios
}

\author{
Carolina Stefoni Espinoza \\ Universidad Alberto Hurtado, Santiago, Chile/ Centro COES CONICYT FONDAP \\ 15130009 \\ Email: cstefoni@uahurtado.cl
}

\begin{abstract}
Menara Lube Guizardi
IDAES-UNSAM, Buenos Aires, Argentina/Universidad de Tarapacá, Arica, Chile

Email: menaraguizardi@yahoo.com.br
\end{abstract}

\author{
Herminia Gonzálvez Torralbo \\ Universidad Central de Chile, Santiago de Chile, Chile \\ Email: herminiagonzalvez@gmail.com
}

\begin{abstract}
Resumen: El presente artículo discutelas políticas de control hacia los movimientos migratorios y la producción de población migrante destinada a "suplir” mercados laborales precarizados. Utilizamos como ejemplo analítico el caso de Chile y las medidas implementadas en 2018 por el gobierno nacionalhacia la migración venezolana y haitiana.Analizamosde qué modo el proceso de fronterización permite comprenderlos ejercicios de delimitación cultural e identitaria de la nación, y la construcción de sujetos cuya inserción económica y social queda condicionada a espacios de vulnerabilidad. Para ello, presentaremosun estado del arte respecto del concepto de fronterización ycaracterizaremos las principales políticas y medidas adoptadas por el gobierno de Chile hacia los flujos migratorios provenientes de Venezuela y Haití. Finalmente, entregamos las principales conclusiones que se derivan de estos casos, en particular los efectos de las medidas de control y gestión migratoria en la construcción diferenciada y selectiva de estos movimientos.

Palabras clave: Migraciones, fronteras, régimen global de migraciones, América Latina.
\end{abstract}

\section{The political construction of the border. Between nationalist discourses and the "production" of precarious workers}

Abstract: This article discusses migratory movement control policies and 
the production of migrant populations destined to "supply" precarious labor markets. We use as an analytical example the case of Chile and the measures implemented in 2018 by the national government towards Venezuelan and Haitian migration. We analyze how the process of borderization allows us to understand the exercises of cultural and identity delimitation of the nation, and the construction of subjects whose economic and social insertion is conditioned to spaces of vulnerability. To do so, we will present a state of the art regarding the concept of border and characterize the main policies and measures adopted by the Chilean government towards migratory flows from Venezuela and Haiti. Finally, we deliver the main conclusions arising from these cases, in particular the effects of migration control and management measures on the differentiated and selective construction of these movements.

Keywords: Migration, borders, global migration regime, Latin America.

\section{A construção política da fronteira. Entre discursos nacionalistas e a "produção” de trabalhadores precários}

Resumo: O presente artigo discute as políticas de controle aos movimentos migratórios e a produção de população migrante destinada a "suprir” mercados laborais precarizados. Utilizamos como exemplo analítico o caso de Chile e as medidas implementadas em 2018 pelo governo nacional em relação a migração venezuelana e haitiana. Analizamos de que modo o processo de fronteirização permite compreender os exercícios de delimitação cultural e identitária da nação, e a construção de sujeitos cuja inserção econômica e social está condicionada a espaços de vulnerabilidade. Para isto, apresentaremos um estado da arte sobre o conceito de fronteirizacao e caracterizamos as principais políticas e medidas adotadas pelo governo do Chile em relação aos fluxos de Venezuela e Haiti. Finalmente entregamos as principais conclusões que se derivam destes casos, em particular os efeitos das medidas de controle e gestão migratória e a construção diferenciada e selectiva destes movimentos. Latina

Palavras chave: Migração, fronteiras, regime migratório global, América

\section{Introducción}

El crecimiento de la migraciónintrarregional en América Latina (Martínez y Orrego, 2016)deja al descubierto una tensión que se agudiza y se vuelve más crítica conforme aumenta el número de migrantes en las ciudades latinoamericanas y avanzan las políticas neoliberales que hacen tambalear los incipientes derechos y las seguridades sociales alcanzados en décadas anteriores por estos mismos países. Esta tensión, que en algunos países asoma como cierta incomodidad, distancia o incluso como abierto rechazo a determinados grupos de migrantes, adquiere su expresión más institucional en el diseño de las políticas migratorias que buscan, a través de las leyes, decretos, acuerdos, normas y programas, dificultar el ingreso de ciertos grupos, condicionar algunos derechos en función del tiempo de estadía en el país receptor y generar mecanismos que precarizan las condi- 
ciones de residencia. Ejemplo de lo anterior son los permisos temporales de trabajo, permisos para dependientes que no permiten trabajar, demoras excesivas en otorgamiento de documentos, entre otros.

No es un misterio para nadie que a nivel global las legislaciones migratorias de los principales países receptores comenzarona cerrar las fronteras nacionales frente a determinados flujos migratorios que comenzaron a ser considerados peligrososo indeseados por los Estados y gobernantes pese a la creciente necesidad de trabajadores migrantes que estos mismos países requieren debido a las transformaciones sociodemográficas que experimentan desde hace décadas-. Los atentados a Nueva York en 2001 imprimieron mayor velocidad a estas medidas que ya venían implementándose, reforzando las políticas de control y seguridad tanto dentro como fuera de los límites territoriales del Estado nación. Lo novedoso hoy en día es que este tipo de enfoque ha comenzado a ser adoptado también por aquellos países menos desarrollados pero que han visto un crecimiento relativo en el número de personas que cruzan sus fronteras en busca de nuevas oportunidades de vida.

Las restricciones a la migración no son consecuencia ni responden al incremento en el número de migrantes en los distintos países receptores, sino más bien se relacionan directamente con la conjunción de dos elementos centrales. Por una parte, la implementación de políticas que buscan garantizar el desarrollo de la economía global neoliberal, a través, por ejemplo, de medidas que buscandisminuir y suspender los sistemas de protección social y que flexibilizan el empleo, con el consecuente proceso de precarización de las condiciones laborales (Anderson, 2010; Benencia, Lima, y Levine, 2012). Por otra parte, el despliegue y reproducción de discursos nacionalistas que intentan instalar la idea de que la migración se ha vuelto una amenaza a las identidades y a las formas de vida moderna de los países desarrollados (Appadurai, 2007). Por lo tanto, las políticas de cierre y control que se han venido implementando desde hace algún tiempo en prácticamente todos los países actúan y tienen efecto en estas dos dimensiones. En primer lugar, tienen la capacidad de producir a sujetos migrantes en condiciones de vulnerabilidad extrema, lo que los transforma en candidatos perfectos para los mercados laborales precarizados, extensivos aún en mano de obra, y que no requieren trabajadores con calificación (aunque muchos de los migrantes que terminan insertándose allí, tienen calificación necesaria para empleos de mejor calidad). En segundo lugar, las políticas de control se presentan como una "solución" frente a la idea construida de amenaza que sostienen los discursos nacionalistas, ganando con ello importantes votos de la población local que ha visto cómo sus condiciones de vida disminuyen año a año debido a los procesos de desindustrialización, apertura a los flujos globales del capital financiero especulativo y precarización del mercado laboral.

En este artículo nos proponemos analizar las políticas migratorias adoptadas por el gobierno de Chile hacia la migración proveniente de Venezuela y de Haití, a partir del concepto de fronterización. Con ello buscamos 
dar cuenta de cómo estas medidas han logrado - en el caso haitiano- disminuir el ingreso de migrantes, favorecer discursos nacionalistasy producir cuerpos racializados y desterritorializados que resultan imprescindibles en el mercado de trabajo. En el caso venezolanoestas medidas han generado demoras ydificultades en el ingreso y en el proceso de regularización producto de una serie de requisitos solicitados por el gobierno de Chile que resultan muy difíciles de obtener.

Este análisis nos permitirá, a su vez, plantear algunas hipótesis respecto del giro que ha adoptado la política migratoria en el país en los últimos años, a partir de la relación entre seguridad nacional, protección de derechos humanos y desarrollo de las economías locales. Este giro estaría fortaleciendo instrumentos para la selectividad migratoria (a través del control y gestión migratoria) y produciendo una inserción laboral diferenciada de los migrantes en los países receptores.

Para ello,la primera parte entrega una discusión y estado del arte respecto del concepto de fronterización. La segunda analiza la relación entre una política liberal y unade mayor control y selectividad en el marco de la migración sudamericana. La tercera parte desarrolla una breve caracterización de las principales políticas y medidas adoptadas por el gobierno de Chile hacia los flujos migratorios provenientes de Venezuela y Haití y también ofrece algunos antecedentes respecto de medidas similares implementadas por otros gobiernos de países de la región. Finalmente, entregamos las principales conclusiones que se derivan de estos casos, en particular los efectos de las medidas de control y gestión migratoria en la construcción diferenciada y selectiva de estos movimientos.

\section{Proceso de fronterización. Elementos para el análisis}

El estudio de las fronteras ha recorrido un largo camino a través de distintos enfoques teóricos y diversas disciplinas -desde la historia, pasando por la política, la geografía, la antropología y la sociología- (ver Kolossov, 2005). Desde el siglo XIX hasta mediados los años cincuenta, el enfoque estuvo dominado por una comprensión fija y estática de la frontera que hizo parte de estudios históricos y geopolíticos que la representaban como líneas divisorias entre Estados-naciones (Brenna, 2011). Es decir, se la asumía como una línea no problematizada, como algo dado que marcaba el límite físico, cultural y político entre las sociedades modernas (ZapataBarrero, 2013). Esta forma de concebir la frontera estuvo presente también en la investigación vinculada con la teoría funcionalista en los cincuenta y sesenta, desde donde se observaron los espacios de interacción e intercambio transfronterizo, así como el rol de las fronteras en los conflictos internacionales (Prescott, 1978). Así, durante parte importante del siglo XX las fronteras fueron comprendidas casi exclusivamente desde su dimensión territorial, de manera descriptiva, sin problematizar su construcción (Grimson, 2011), lo que sin duda contribuyó al discurso homogeneizante y naturalizado del Estado-nación (Wimmer \& Glick Schiller, 2003). 
A partir de los setenta se observan nuevos abordajes y perspectivas que permitieron abrir esta categoría, favoreciendo el desarrollo de una serie de investigaciones situadas en distintos puntos del planeta (Sum, 2003). La influencia de la teoría del sistema mundo (Wallerstein, 1995), el posterior giro espacial en los estudios urbanos (Appadurai, 1998; Harvey, 2003; Lefebvre \& Levich, 1987), el desarrollo de la perspectiva transnacional en los estudios migratorios (Bash, Glick Schiller, \& Szanton Blanc, 1994), y el análisis desde la dimensión cultural respecto del proceso de globalización e identidades (Appadurai, 1998; García Canclini, 1990)permitieron comprender las fronteras como un elemento clave en la organización de un sistema económico global (Sum, 2003), sustentado en dependencias y jerarquizaciones estatales (Mezzadra \& Neilson, 2013) herederas de un orden colonial.

Por otra parte, utilizando una aproximación constructivista -y principalmente desde la antropología- se desarrollaron análisis de los significados simbólicos y subjetivos de quienes experimentaban una frontera que comenzaba a ser más resguardada y controlada (Garduño, 2003; Velasco y Contreras, 2011). Abordarla frontera como un proceso permitió comprender las distinciones colectivas de los grupos sociales, sus identificaciones y la distribución de los rasgos culturales (Barth, 1976), transformándolas en un locus para analizar la interacción de las dimensiones cultural, política y social en la configuración del Estado-nación (Kearney, 1991; Vila, 2000). A su vez, proveyeron de un eje clave para observar diferencias y desigualdades articuladas en torno a las categorías de nacionalidad y etnia (Grimson, 2004; Guizardi, Valdebenito, López, y Nazal, 2015; Kearney, 2004).

El desarrollo actual de los estudios de frontera y los estudios críticos de frontera (border studies y critical border studies) han permitido problematizar la categoría poniendo en el centro del debate las diversas relaciones de poder político, simbólico y social sobre las que estas se construyen (Rumford, 2006). A su vez, da cuenta de la complejidad para definirla en la medida en que pretende delimitar los contornos territoriales de los propios Estados que la definen (Mezzadra \& Neilson, 2013). Tal como nos recuerda De Génova (2013), finalmente son las fronteras las que crean la migración y las que permiten la existencia de la ciudadanía.

Estas importantes contribuciones permitieron desterritorializar la frontera (al situar el análisis en la gobernabilidad y la biopolítica) y comprender su uso en cuanto elemento articulador del orden global neoliberal. Esta aproximación permite trascender la distinción binaria adentro/afuera, inclusión/exclusión propia de la comprensión lineal y territorial de la frontera (Kearney, 2004; Sum, 2003), planteando que se trata de una inclusión a través de la exclusión/ilegalización producida por las políticas de control que operan dentro de la herencia del sistema colonial (De Genova, 2015). En un registro equivalente Mbembe señala (en De Génova, 2015) que el racismo es la clave para entender la inclusión de migrantes como trabajadores subordinados, ya que la condición de esclavos es resultado de una triple pérdida: de su hogar, de sus derechos y de su cuerpo. 
La desterritorialización a su vez problematiza la imagen de muro divisorio frecuente en los estudios sobre seguridad (Vallet, 2014). Al pasar del estudio de la frontera al estudio sobre el proceso de fronterización (ZapataBarrero, 2013), observamos cómo esta se transforma en la herramienta con que cuentan los Estados y los mercados para diferenciar y jerarquizar los movimientos de personas (Amoore, 2013): turistas de potenciales trabajadores; businessman de posibles refugiados, trabajadores calificados de trabajadores para la agricultura y la construcción, trabajadoras en el comercio sexual de víctimas de trata y tráfico. La frontera nunca es un cierre absoluto, sino un ejercicio que transforma la movilidad de personas en política para decidir cómo y quiénes pueden efectivamente moverse (De Genova, 2015).

De este modo, la diferencia entre el régimen de control global actual y los mecanismos de control en fronteras que han existido desde hace mucho tiempo atrás, radica enque hoy asistimos a unaarticulación entre los procesos de fronterización, el orden económico y el sistema político (Vila, 2000). El incremento en las prácticas de violencia(política y simbólica) se transforma de este modo, en la herramienta que permite asegurar esta articulación. El discurso que legitima el fortalecimiento de este régimen de control y uso de la violencia, es paradójicamente la defensa de los derechos de los propios migrantes (Mezzadra \& Neilson, 2012), así como la seguridad de los nacionales de los países receptores. Este régimen de control y de frontera se observa y dibuja no sólo en las interacciones que se producen en los límites geográficos entre un Estado y otro, sino también a través de una serie de artefactos, discursos, políticas y medidas instaladas desde el centro nacional que se ponen en práctica en diversas ciudades del territorio, en las regiones de fronteras e incluso más allá de los límites territoriales de los Estados (Amoore, 2013; Bigo, 2015; De Genova, 2013).

La reflexión en torno a las fronteras que realiza Balibar (2010) adquiere, entonces, todo su sentido:

"Las fronteras no son nunca sólo instituciones locales, nunca se pueden reducir a la simple historia de conflictos y acuerdos entre grupos de vecinos y de poder. Las fronteras son globales, son formas de dividir el mundo en regiones posibilitando la emergencia del espacio y los mapas imaginarios. Las fronteras se caracterizan por una ambivalencia interna que deriva de su función interna y externa: la pertenencia al colectivo y el control estatal sobre la movilidad y el territorio. La construcción del espacio político ocurre a través de modos de traducción entre el adentro y afuera, que es significado por las fronteras”(Balibar , 2010, p. 316)(Traducción propia).

En América Latina, la frontera norte México-USA ha entregado innumerables herramientas conceptuales y metodológicas que sirvieron de marco analítico (Kearney, 1991, 2004; Vila, 2000). Sin embargo, en la medida en que avanzaron estos otros estudios, se hizo evidente las singularidades que aportaban y la necesidad, por ende, de complejizar los marcos conceptuales utilizados (Grimson, 2005). En Chile, los estudios de fronteras se 
concentran en el norte del país. Tapia introduce el concepto de regiones fronterizas para incorporar una mirada espacio-temporal en su configuración (Tapia y Parella, 2015;Tapia y Ovando, 2013); otros autores analizan la relación entre fronteras y Estado-nación (Dilla, 2016; S. González, 2009); la relación entre migración y criminalización como estrategia de fronterización (Dufraix y Quinteros, 2017; C. Norambuena, 2013; Quinteros, 2016); el mundo andino y su relación con las fronteras nacionales (Bello, 2012; H. González, 1998); los desafíos del género en la construcción de fronteras (Guizardi, 2016; Guizardi y Garcés, 2012). Desde miradas heterogéneas, estos estudios contribuyeron a conceptualizar las fronteras como espacios plurales donde los distintos Estados-nación actúan estructuralmente (construyendo legitimidades y regímenes de adscripción de las gentes), mientras la agencia de sujetos diversos (a través de los desplazamientos) resignifica y negocia las clasificaciones nacionales (Brenna, 2011, p. 12). Según estos debates, el enfoque analítico para trabajar fronteras debe, al mismo tiempo en que capta la persistencia y reproducción social las categorías estáticas acerca de las pertenencias nacionales, observar su rearticulación a partir del movimiento y porosidad que caracterizan a los espacios fronterizos. Una segunda línea de investigación desarrollada en Chile y que resulta de gran relevancia, es el análisis de la política migratoria, tanto en un registro histórico como en elanálisis actual (Doña y Mullan, 2014; Durán y Thayer, 2017; Norambuena Carrasco, 1990; Stefoni, 2011; Thayer, 2016).

Así, el debate chileno sobre las fronteras se viene acercando a las discusiones llevadas a cabo por investigadores de otros territorios de frontera en Sudamérica. Autores como Grimson (2000) señalan que la porosidad de las negociaciones sociales en las fronteras no implica modificaciones de las "clasificaciones identitarias y autofiliaciones nacionales. Más bien, es sobre la existencia de la frontera que se organiza un sistema social de intercambios entre grupos que se consideran distintos”(p.28). Esta reflexión sedimenta la consideración de que las disparidades jurídicas, políticas, económicas e identitarias de espacios nacionales colindantes provocan la emergencia de prácticas sociales que buscan beneficiarse de estas diferencias (Grimson, 2005) a partir de provocar una liminaridad de la relación entre licito e ilícito y entre pertenencia y desarraigo. Históricamente, estas prácticas han usado la movilidad y circularidad transfronteriza como un mecanismo fundamental para lograr estos beneficios e intereses (individuales, grupales o colectivos) (Cardin, 2012). En el caso de las fronteras chilenas, estas reflexiones se verifican en los trabajos de Amilhat (2007), González (2006, 2009), Guizardi y Garcés (2013), Guizardi et al (2017), Tapia (2012, 2015), Tapia y Ramos (2013).

\section{¿Políticas liberales o selectivas en materia migratoria?}

América Latina ha experimentado un importante crecimiento en los movimientos migratorios intrarregionales. Al profundizar en los factores que explican el crecimiento de la migración regional, la literatura menciona: i) la emergencia de algunos destinos atractivos por su estabilidad económi- 
ca y política, como es el caso de Chile y Costa Rica; ii) la incidencia de algunas medidas migratorias tomadas en el marco de convenios de integración regional, como el Acuerdo de Residencia de Mercosur, que facilitan la circulación de personas entre los países de América del Sur (Texidó \& Gurrieri, 2012); iii) la cercanía entre los países (Mora y Undurraga, 2013); iv) las transformaciones y apertura de los mercados laborales; v) el menor costo en transporte (Martínez y Orrego, 2016); vi) las crisis humanitarias específicas, como es el caso de Haití (OIM, IPPDH, \& MERCOSUR, 2017; Rojas et.al, 2017; Vásquez et.al, 2015); vii) la crisis económica y política en Venezuela (Freitez, 2011; International Organization for Migration OIM, 2018) y, finalmente, viii) el endurecimiento de las políticas de control y medidas restrictivas en países del norte global.

Los datos de las rondas censales indican que América del Sur congrega a los países con saldos migratorios más bajos de América Latina (proporción entre la población que emigra y la que inmigra). Sin embargo, en los últimos años creció de manera importante la migración intrarregional (personas nacidas al interior de la región o fuera de esta y que se encuentran viviendo en otro país latinoamericano), aunque Estados Unidos sigue siendo el destino principal para los migrantes de estos lares. Este crecimiento no es independiente de la agenda regional que promueve y favorece la circulación de personas, como tampoco lo es de los cambios y adaptaciones que realizan las economías locales para insertarse en la economía global.

El principal instrumento con que cuenta América del Sur para promover la movilidad de trabajadores es el acuerdo de Residencia de Mercosur. Este acuerdo permite a cualquier nacional de los Estados miembros plenos o "naciones amigas", obtener una residencia temporal en el país de la subregión al que emigre, independiente de la tenencia de contratos laborales. Tanto UNASUR como la Conferencia Sudamericana de Migraciones manifestaron su apoyo a este acuerdo y declararon la voluntad de seguir avanzando en la integración regional. Incluso antes de que algunos países comenzaran a suspender su participación en la UNASUR (Argentina, Brasil, Chile, Colombia, Paraguay y Perú), el organismo llegó a plantear la idea de desarrollar una ciudadanía mercosureña. El acuerdo de residencia fue implementado en los distintos países de la región (con diferencias notables entre unos y otros) (Modolo, 2010). Argentina, por ejemplo, incluyó el principio de residencia en su constitución y hoy lo sigue aplicando incluso para los venezolanos que han llegado a ese país (Venezuela ha sido suspendida temporalmente del acuerdo). Chile es el único país que lo aplica sólo por acuerdo bilateral con 5 países (Argentina, Brasil, Uruguay, Paraguay y Bolivia). El desarrollo de esta agenda de integración da cuenta de la presencia de un discurso liberal que ve positivamente la migración y que promueve la protección de los derechos humanos de los migrantes.

Ahora bien, ¿qué ha empujado a Mercosur a promover la circulación de personas en una región que se caracterizaba, al menos en el inicio del acuerdo, por un bajo nivel de migración intrarregional? Si estamos de acuerdo en que la integración económica ha sido uno de los principales objetivos 
de Mercosur, la baja movilidad de personas constituye un "problema” en el proceso de adaptación de las economías locales a la economía global. Las economías desarrolladas del mundo encontraron en la movilidad de personas una fuente de trabajadores flexibles y adaptables para los requerimientos de la nueva economía (Anderson, 2010; Bolch, Fellini, Ferro, Fullin, \& Hunger, 2004; Castells, 2004; Munck, 2012). La apertura de las economías mercosureñas al mercado global requería entonces, entre otras cosas, de trabajadores que pudieran cruzar las fronteras e insertarse rápidamente a las nuevas -y flexibles-demandas del mercado laboral. Así, el incentivo a la migración a través de los acuerdos de residencia y apertura de fronteras son estrategias políticas que favorecen dinámicas económicas específicas. Por otra parte, existe una serie de proyectos para la conectividad bioceánica en la región (Grimson, 2005, p. 90), cuestión que se ha vuelto el corazón geoestratégico del MERCOSUR (Sausi y Oddone, 2010). Estos megaproyectos garantizan grandes inversiones de dinero, dinamizan el mercado laboral y promueven cierto tipo de integración regional.

En definitiva: los saldos migratorios bajos implicaban una baja capacidad de adaptación a la lógica circulatoria transnacional y transfronteriza que caracteriza la gestión de las actividades económicas en la globalización y en la postglobalización. El discurso liberal buscó revertir esta situación favoreciendo la movilidad de personas (no de todos, por cierto) entendiendo que se trata de un factor clave para el desarrollo de las economías. En el caso específico de América del Sur, este discurso liberal estuvo acompañado de una narrativa importante de defensa de los derechos humanos de los migrantes(Domenech, 2009; 2013). De hecho, en diversas instancias de diálogo birregional (EULAC, Foro Iberoamericano) y acuerdos regionales en torno a las migraciones, se ha enfatizado el compromiso con la defensa de los derechos humanos de las personas migrantes y el rol de los Estados en esta protección (CIDH, 2015; Texidó y Gurrieri, 2012).

Sin embargo, en los últimos tres años se puede identificar un giro en la política migratoria subregional (Acosta y Freier, 2015). En 2015, Acosta y Freier se preguntaban si este discurso liberal y de protección de derechos era efectivo, es decir, sustentado en legislaciones, programas y políticas orientadas a la protección de los derechos de las personas migrantes, o bien se trataba de un discurso liberal que promovía la apertura, pero con restricciones a determinados colectivos, y condicionamientos a los derechos en función del estatus migratorio y tiempos de residencia, entre otros. Diversos autores analizan y sostienen que el cierre de fronteras en Europa (Andrijasevic, 2010), Norteamérica (De Genova, 2018; Kretsedemas \& Brotherton, 2018) y en la mayoría de los estados con economías desarrolladas, es de carácter selectivo (se le impide la entrada a nacionales de determinados países) y funcional (al ritmo y demandas de los mercados laborales) (Anderson, 2010; Appadurai, 2007; Bauman, 2016; Castells, 2004; Mezzadra, 2016; Salazar Parrenas, 2000). Pero de ser cierto, el giro hacia políticas de mayor control en América del Sur (que consideramos sí lo es), ¿se trata sólo de una incorporación y adaptación de lo que ocurre hoy día en los países del centro? ¿Tiene alguna lógica y dinámica propia? y ¿Qué 
implicancias tendría esta tendencia en una región que se percibe semiperiférica dentro del orden global?

El giro hacia una política de mayor control no significa que se haya pasado de un discurso de integración a uno opuesto de control y seguridad, sino que se trata más bien de un cambio en la relación de fuerzas entre los dos principios que fundan la política migratoria: los derechos humanos y la seguridad nacional (De Lucas, 2003). Siguiendo el estudio que realiza Domenech para el caso de Argentina (Domenech, 2009), hipotetizamos que en el discurso de integración desarrollado durante la primera década del 2000, ya existían ciertos elementos que condicionaban, restringían y relativizaban los derechos que el discurso liberal reconocía a la población migrante. Todo esto implica nuevos regímenes de fronterización de ciertos colectivos migratorios en la región. Nos detendremos en dos ejemplos que han ganado relevancia en los últimos años: el de los y las migrantes de Venezuela y Haití.

\section{Venezuela y Haití: selectividad y cierre como mecanismos de fronterización}

Venezuela ha sido históricamente uno de los países junto con Argentina que más migrantes recibieron en la región. Más de un millón de personas fueron atraídas durante décadas por una economía sustentada en la industria petrolera (Chi Yi et al., s. f.). Sin embargo, la crisis del modelo económico extractivista, elevados índices de violencia y la inestabilidad política de los últimos años generaron las condiciones para un proceso emigratorio que alcanza hoy día proporciones de una crisis humanitaria (Freier, 2018). Los primeros destinos fueron Estados Unidos y Europa: de los 432.000 migrantes registrados en la ronda de censos 2010, había 185.000 venezolanos residiendo en Estados Unidos y 155.000 en Europa (Stefoni, 2018a). Pero la situación ha cambiado en los últimos cinco años. Se estima que entre 1.500 .000 a 4.000.000venezolanos se encuentran fuera de su país, situación que se acentuó en 2017 de acuerdo con medios de prensa, organizaciones sociales e informes internacionales. La crisis económica reflejada en una elevadísima inflación, escasez de alimentos, remedios y bienes cotidianos constituye actualmente la primera causa de salida. El empobrecimiento de la sociedad venezolana sólo se entiende si se toma el peso de lo que implica que más del $80 \%$ de la población se encuentre actualmente bajo la línea de la pobreza.

El éxodo de venezolanos se ve reflejado también en el número de solicitudes de asilo a nivel global. Si en 2014 se contabilizaban 4.040 solicitudes de asilo, en 2017 fueron 94.284 (UNHCR, 2018). La información disponible da cuenta de que más de la mitad de la diáspora venezolana reside actualmente en Sudamérica. El informe de la OIM señala que del total de emigrantes venezolanos en el mundo (1.622.109, cifra aún conservadora de acuerdo a otros informes), 885.891 se encontrarían en algún país de América 
del sur (OIM, 2018). En todos los casos, destaca el alto nivel de educación que poseen los migrantes (Freier, 2018), aunque pareciera ser que cada vez se migra con menos recursos económicos.

Los países de la región han respondido de diversas maneras a esta situación (Freier, 2018): Argentina y Uruguay han aplicado la visa de Residencia Mercosur, pese a que Venezuela tiene su estatus suspendido en dicha instancia. Esta visa permite residir legalmente por un período de dos años. Perú otorga, desde enero de 2017, el Permiso Temporal de Permanencia. Empero, su aplicación ha sido problemática, puesto que los que efectivamente se han cursado son mucho menos que las solicitudes realizadas (35.000 de 350.000, el 10\%). Esta situación ha llevado a que muchos soliciten refugio como una forma más expedita para obtener la residencia. Brasil también optó por crear un permiso especial para venezolanos que otorga residencia de dos años, similar a la visa Mercosur.En Chile, el presidente Piñera implementó, en abril de 2018, una visa democrática para los ciudadanos de dicho país, en el marco de una serie de anuncios de medidas específicas en materia migratoria.

Con fecha 9 de abril de 2018 el Presidente Sebastián Piñera ingresó un proyecto de Ley de Migraciones que busca terminar con el Decreto Ley $\mathrm{N}^{\mathrm{o}} 1094$, de 1975 promulgado en dictadura, con una orientación basada en la seguridad nacional y que de acuerdo a todos los sectores, no permite dar respuesta a la actual realidad migratoria (Tapia y González, 2014; Thayer, 2015). El proyecto presentado en 2018 recoge gran parte del que fue presentado en la primera administración de Piñera, en 2013 (Stefoni y Silva 2018).

Junto con el ingreso del proyecto de Ley al parlamento, el Presidente anunció una serie de medidas administrativas orientadas a "ordenar la casa", tal como él mismo lo indicó, adelantando con ello algunos de los lineamientos considerados en el proyecto, de modo de poder comenzar con su implementación independiente del curso que tome la discusión del en el Congreso. Estas medidas fueron las siguientes:

1. Reformulación del sistema de visados: elimina la posibilidad del visado temporario por motivos laborales (creado en 2015) a contar del 23 de abril de 2018; crea por vía administrativa una visa Temporaria de Oportunidades, que debe ser solicitada fuera de Chile, está orientada a todos aquellos que quieran viajar a Chile y otorga un permiso para residir y trabajar por un año, prorrogable a otros 12 meses; crea una Visa Temporaria de Orientación Internacional para personas con posgrados en alguna de las mejores universidades del mundo, según ranking que se establecerá para dicho efecto. La visa es por un año prorrogable.Crea Visa Temporaria de orientación nacional posible de solicitar en Chile y que será otorgada automáticamente a migrantes que obtengan posgrado en universidades chilenas acreditadas. Se otorga por un año, prorrogable por un segundo. Estas visas comenzaron a regir a partir del 1 de agosto de 2018. 
2. Se determina la exigencia de un visado consular de turismo simple por un período máximo de 30 días a ciudadanos haitianos. Se establece un visado humanitario de reunificación familiar. Se entregarán 10 mil visados humanitarios al año.

3. En el caso de Venezuela, se crea una Visa de Responsabilidad Democrática a ser solicitada en dicho país y que otorga un permiso de residencia temporal por un año, prorrogable por una vez.

4. Inicio de un proceso de regularización para todos aquellos que se encuentran en situación irregular.

Los anuncios del Presidente Piñera sorprendieron a las organizaciones sociales pues significaba un endurecimiento en el enfoque hacia la migración. La visa consular a haitianos era un claro indicio de la apuesta por restringir este flujo de personas.

En el caso de la visa de responsabilidad democrática fue bien recibida por la población venezolana que estaba con la intención de emigrar a Chile. La posibilidad de obtener rápidamente un visado y estar en situación regular al momento de migrar, constituía una muy buena noticia. Sin embargo, en un comienzo hubo serios problemas de información y desconocimiento por parte del consulado en Caracas y en Puerto Ordaz. En blogs de opinión se reconocía una lenta implementación y desconocimiento inicial por parte de estosconsulados. La desinformación llevó a que se confundieran las medidas decretadas por Piñera y que se interpretara como que la única posibilidad para emigrar a Chile era a través de esta visa democrática, siendo que subsisten hasta el día de hoy la forma histórica de migrar hacia Chile (ingreso como turista y solicitud de cambio de visa dentro del país), cuestión que continuará operando así hasta que se modifique la Ley.

Con todo, a un mes de implementarse, y de acuerdo a información entregada por los medios de prensa, se habían ingresado 20.638 solicitudes (incluye todos los ingresos, ya sea con errores o duplicaciones, por los que las solicitudes efectivas serían menos numerosas). De ellas, se habían entregado 2.131;se habían descartado por falta de antecedentes unas 971 y 151 habían sido rechazadas por no cumplir con los requisitos establecidos por el gobierno de Chile (La Tercera, miércoles 16 de mayo de 2018).El 3 de junio las solicitudes efectivas sumaban 27.910 y se habían aprobado 3.800. Unas 3.200 habían sido rechazadas por falta de antecedentes y 219 rechazadas por no cumplir los requisitos establecidos (Teletrece, 3 junio 2018).

Uno de los principales problemas señalados en entrevistas y blogs son las dificultades para conseguir los requisitos establecidos. Los requisitos iniciales para solicitar esta visa eran: Certificado de antecedentes penales con vigencia de 90 días, debidamente apostillado con código de verificación legible (se exceptúan los menores de edad para este requisito); pasaporte vigente y una fotografía de estudio a color 5 x 5 , fondo blanco. Sin embargo, la información en la cuenta twitter del consulado Chileno en Vene- 
zuela el 21 de junio de 2018 confirmó la inclusión de dos nuevos requisitos: el pasaporte deberá tener una vigencia de 18 meses (no se aceptan pasaportes provisionales), y los menores de edad deberán presentar acta de nacimiento apostillada con su código de validación.

Efectivamente, uno de los mayores problemas para esta visa es la obtención de los certificados y el pasaporte. Las cifras por otra parte indican una alta expectativa por esta visa, pero una baja tasa de aprobación, debido probablemente a las dificultades que tiene el consulado para tramitar todas las solicitudes.

Por otra parte llama la atención la omisión en la propuesta de Ley y en las medidas administrativas, al acuerdo de residencia de Mercosur. Si bien Chile no ha suscrito dicho acuerdo y sólo lo tiene vigente con los países con los cuales ha firmado acuerdos bilaterales (Argentina, Brasil, Uruguay, Paraguay y Bolivia), esta ausencia reitera el escaso interés de la administración actual por ratificar e implementar el acuerdo. Es importante recordar que, en el país, más del 70\% de la migración proviene de América del Sur, por lo que el acuerdo de libre residencia permitirá al 70\% de toda la migración estar en una situación regular desde un inicio (Aguirre et al., 2010).

La migración haitiana representa otra de las movilidades poblaciones complejas en la actualidad. El terremoto de 2010, el brote de cólera que le siguió, dos huracanes que terminaron por devastar la infraestructura y la economía del país, y el cierre de las fronteras en el norte llevaron a esta población a buscar nuevos destinos en la región, más allá de República Dominicana y Estados Unidos.

Brasil fue, en un primer momento, el principal receptor de estos flujos ya sea de quienes venían directamente de Puerto Príncipe, o bien a través de Colombia (Mamed \& Oliveira de Lima, 2017). En 2011 implementó una visa humanitaria que reconoció las condiciones de vulnerabilidad estructural del país de origen (Feldman-Bianco, Cavalcanti, \& Araujo, 2018), como causa suficiente para habilitar la regularización migratoria. Se estableció, además, un cupo anual de 1200 visas, pero la presión los llevó a liberar en 2012 este número (Da Silva, 2017). Perú, a su vez comenzó a solicitar en 2012 una visa consular de turismo a través de un trámite en Puerto Príncipe. Un informe elaborado en Lima por el Instituto de Estudios Peruanos (IEP) sostiene que el alto costo y las dificultades para obtener dicha visa hizo que fueran muy pocos los que efectivamente la solicitaron (Vásquez et al., 2015). Argentina no solicita visa de turismo y emitió, en 2017, una resolución para regularizar a aquellos migrantes que hubiesen ingresado como turistas antes del 1 de marzo de ese año (Disp. 1143-E/17). Chile, en abril de 2018, estableció una visa para el ingreso como turistas, cerrando con ello la posibilidad de migrar como turistas y cambiar el estatus migratorio una vez dentro (como lo hacen actualmente todas las otras nacionalidades). Los requisitos para la visa consular son difíciles de conseguir: pasaporte vigente por el período de estadía; certificado de antecedentes (legalizado) con 
vigencia de 90 días; reserva de hotel o carta de invitación; copia de pasajes comprados (ida y vuelta) y acreditación de solvencia económica (la escasa bancarización que existe en Haití hace bastante difícil acreditar esta solvencia). En este contexto, no llama la atención el bajo número de solicitudes que se han cursado (Stefoni, 2018b). Si a ello le sumamos el reducido cupo de visas por reunificación familiar, y la dificultad para migrar con la visa de oportunidades (creada para personas altamente calificadas y emprendedores que cuenten con varios miles de dólares), la política migratoria chilena hacia Haití más se asemeja a lo hecho por Europa con Siria, que al llamado a proteger los derechos humanos de personas que se encuentran en una situación crítica.

\section{Conclusiones: La producción de fronterizaciones y de las crisis humanitarias migrantes}

Los complejos escenarios migratorios de Haití y Venezuela nos conducen hacia dos consideraciones analíticas. En primer lugar, si las categorías de migración y refugio grafican realmente el alcance que tienen estos movimientos, es decir, ¿permiten responder a la situación que enfrentan las personas que salen de dichos países? En segundo lugar, ¿qué significa “crisis humanitaria”? La violencia que expulsa, así como la violencia que marca el recorrido por una América Latina cada vez más indolente, y la violencia que se experimenta en los lugares donde se intenta sacar adelante proyectos individuales, familiares y colectivos corren a la par de la implementación de nuevos mecanismos de control migratorio, cierre selectivo de fronteras y la implementación de discursos de criminalización y rechazo de los migrantes. En este sentido,consideramosque la crisis humanitaria está dada por un sistema de violencia cuya expresión máxima es la búsqueda desesperada de una puerta de entrada a algún país que les permita encontrar algo de tranquilidad. Es, por tanto, el cierre selectivo de fronteras, amparado en una lógica de gestión y gobernanza migratoria, lo que genera la situación de “crisis humanitaria” (que quizás esté mejor descripta como una crisis de humanidad).

La política migratoria de un país como Chile debe ser analizada dentro de lo que se ha planteado como un cambio en el paradigma liberal de la política migratoria y de asilo en América Latina (Acosta y Freier, 2015; Ceriani, 2011; Mármora, 2010). Este cambio encuentra en la idea de gobernanza y gestión migratoria una herramienta clave que permite mantener el discurso positivo hacia la migración (discurso liberal), a la vez que introducir mecanismos de control y seguridad nacional (bajo un nuevo régimen de fronterización). Bajo el lema de “migración segura, ordenada y regular” se incrementan los controles migratorios en fronteras(González, 2010; Grimson, 2005; Sausi y Oddone, 2010) (y su militarización, como se observa en el norte de Brasil y en el norte de Chile, con el Programa Frontera Segura); aumentan las deportaciones; se incrementa la negación de ingreso en las fronteras; se crean visas especiales que generan altas expectativas (pero 
con poca materialización); se introducen sistemas biométricos de control no sólo en las fronteras, sino disponibles en cualquier momento y lugar; se perfeccionan los sistemas de registros y se promueve el uso compartido entre países de estas bases de datos. Todo ello bajo el argumento de bienvenida a todos los migrantes que contribuyan al desarrollo de las naciones (y el consecuente rechazo a los que no); y la afirmación de que los dispositivos de control son necesarios para resguardar la seguridad de los propios migrantes.

Pero la complejidad de este contexto no está dada sólo por los cambios en la forma de conceptualizarlas fronteras, sino también por la tensión que enfrenta el derecho a migrar con la soberanía de los Estados para definir quién pertenece o no a la comunidad nacional (De Lucas, 2003; Ghosh, 2008; Kearney, 2004). Algunos autores plantean que esta tensión ha estado a la base de las políticas de control desde la configuración del Estadonación (De Genova, 2013; Domenech, 2009), mientras otros ponen el acento en el giro actual que toma la política de seguridad a partir de una serie de eventos o hitos como la crisis del petróleo en 1973 (Castles \& Miller, 2007), el ataque a las torres gemelas y el pentágono en 2001 (Sørensen \& Gammeltoft Hansen, 2013) y la crisis económica de 2008 (Sanahuja, 2017). Pero estos autores tienen en común un punto articular con el que coincidimos, al menos en el abordaje que creemos necesario para los procesos observados en Chile. Este punto articulador refiere a la necesidad de observar las nuevas fronterizaciones como un recurso político complejo, que requiera que focalicemos a la gubernamentalidad como herramienta analítica (Domenech y Pereira, 2017).El abordaje de estas nuevas formas de fronterización -tal como observamos en el caso de los venezolanos y haitianos en Chilerequiere combinar el análisisde la gestión de la movilidad (Zapata-Barrero, 2013), con el estudio del uso instrumental de bases de datos, de la exigencia de visas y de las biotecnologías para el control de los cuerpos migrantes (Bigo, 2015; Salter, 2016). Requiere, además, abordar el rol de los organismos internacionales en la incorporación de un discurso de gobernanza migratoria para legitimar las restricciones a la movilidad humana (Andrijasevic \& Walters, 2010) y la relación entre gobernanza migratoria y las transformaciones del mercado laboral (Piper, 2017). Al respecto, Piper señala(en Karakayali, 2015) que la migración indocumentada representa el prototipo del trabajador explotado en el sistema neoliberal (carente de derechos, explotable y subordinado). Pero, para que este modelo de explotación "funcione", el Estado también deberá controlar los efectos negativos de esta irregularidad a través, por ejemplo, de separar la residencia de los derechos sociales.Así los migrantes pueden ser autorizados para residir, pero no para tener derechos sociales.

Sostendremos, finalmente, que este proceso de fronterización nos entrega un ángulo privilegiado para observar la emergencia de un nuevo orden global y analizar el lugar que comienza a tener una región intermedia -aunque aún periférica- en este orden. Como diversos analistas vienen señalando (Segato, 1999; Grimson, 2011), las fronteras constituyen un lugar determinante, un locus prioritario para estudiar los procesos de construc- 
ción de los macro modelos políticos y económicos, así como la identidad. En los territorios de frontera, se experimentan las porosidades, conflictos y contactos entre los grupos sociales pertenecientes a uno u otro Estadonación; y la tensión entre flexibilización y reificación de las construcciones identitarias, políticas y económicas.

\section{Agradecimientos}

Centro COES CONICYT FONDAP 15130009 


\section{Bibliografía}

Acosta, D., y Freier, L. F. (2015). Discursos y políticas de inmigración en Sudamérica: ¿Hacia un nuevo paradigma o la confirmación de una retórica sin contenido? Revista Interdisciplinar da Mobilidade $\mathrm{Hu}$ mana - REMHU, XXIII(44), 171-189.

Aguirre, O., Mera, G., y Nejamkis, L. (2010). Políticas migratorias e integracion regional. La libre circulación y los desafíos de la ciudadanía. En S. Novick, G. Mera, \& V. Modolo (eds.), Migraciones y Mercosur: una relación inconclusa (pp. 51-72). Buenos Aires, Argentina: Catálogos.

Amoore, L. (2013). The politics of possibility. Risk and Security Beyond Probability. Estados Unidos: Duke University Press.

Anderson, B. (2010). Migration, immigration controls and the fashioning of precarious workers. Work, employment and society, 24(2), 300-317.

Andrijasevic, R. (2010). From Exception to Excess: Detention and Deportations across the Mediterranean Space. En N. De Genova \& N. Peutz (Eds.), The Deportation Regime: Sovereignty, Space, and the Freedom of Movement (pp. 147-165). Durham: Duke University Press.

Andrijasevic, R., \& Walters, W. (2010). The International Organization for Migration and the international government of borders. Environment and Planning. Society and space, 28, 977-999.

Appadurai, A. (1998). Modernity at large. Cultural Dimensions of globalization. Minneapolis, United States: University of Minnesota Press.

Appadurai, A. (2007). El rechazo de las minorías. Ensayo sobre la geografía de la furia (Primera). Barcelona: TusQuets Editores.

Balibar, E. (2010). At the Borders of citizenship: A Democracy in Translation? European Journal of Social Theory, 13(3), 315-322.

Barth, F. (1976). Introducción. En Los grupos étnicos y sus fronteras (pp. 9-49). Ciudad de México, México: Fondo de Cultura Económica.

Bash, Li., Glick Schiller, N., \& Szanton Blanc, C. (1994). Nations unbound. Transnational projects, poscolonial predicamentsand deterritorialized nation-states.Amsterdam, Países Bajos: Gordon and Breach.

Bauman, Z. (2016). Extraños llamando a la puerta. Barcelona: Paidós. Barcelona, España: Paidós. 
Bello, D. (2012). Alianza estratégica Aymara sin Fronteras: Una respuesta territorial a los desafíos de la «glocalización». Tinkazos. Revista Boliviana de Ciencias Sociales, 32, 147-164.

Benencia, R., Lima, F. F. H.,y Levine, E. (2012). El empobrecimiento del trabajo: vulnerabilidad y precariedad en el mundo del trabajo de la postmodernidad real. En B. Herrera y E. Levine,Ser migrante latinoamericano, ser vulnerable, trabajar precariamente. Barcelona, España: Anthropos/ Ciudad de México, México: Universidad Autónoma Metropolitana

Bigo, D. (2015). Death in the Mediterranean sea. En Y. Jansen, R. Celikates, \& J. De Bloois (Eds.), The irregularization of migration in contemporary Europe. Definition, Deportation, Drowning. London, England: Rowman \& Littlefield International.

Bolch, A., Fellini, I., Ferro, A., Fullin, G., \& Hunger, U. (2004). The political Economy of Labor. Migration in the European Construction Sector. En M. Bommes, K. Hoesch, U. Hunger, \& H. Kolb (Eds.), Organisational recruitment and Patterns of Migration. Interdependencies in an Integrating Europe. Osnabrück, Dinamarca: Osnabrück University.

Brenna, J. E. (2011). La mitología fronteriza: Turner y la modernidad. Estudios Fronterizos, nueva época, 12(24).

Castells, M. (2004). Inmigrant workers and class struggles in Advanced capitalism: The western European experience. En M.,Sadri. (ed.)Migration, Globalization, and Etnics Relations. An Interdisciplinary Approach Mobasher (pp. 250-279). New Jersey, United States: Pearson Prentice Hall.

Castles, S. (2013). Migración, trabajo y derechos precarios: perspectivas histórica y actual. Migración y Desarrollo, 11(20), 8-42.

Castles, S., \& Miller, M. (2007). La era de las migraciones: movimientos internacionales de población. Ciudad de México, México: Universidad Autónoma Metropolitana.

Ceriani, P. (2011). Luces y sombras en la legislación migratoria latinoamericana. Nueva Sociedad, (233).

Chi Yi, C., Urquijo, J., y Picouet, M. (s. f.). Los movimientos migratorios internacionales en Venezuela: Políticas y realidades. Revista sobre Relaciones Industriales y Laborales (10-11)

CIDH. (2015). Derechos humanos de migrantes, refugiados, apátridas, víctimas de trata de personas y desplazados internos: Normas y 
Estándares del Sistema Interamericano de Derechos Humanos (No. Doc 46/15). CIDH OEA.

Comisión Europea, OIM, FIIAPP. (s. f.). Fortalecimiento del diálogo y de la cooperación entre la UE y ALC para el establecimiento de modelos de gestión sobre migración y políticas de desarrollo.

Cotinguiba, M. \& Cotinguiba, G. (2017). Fronteiras e aspectos do rito de mudança de categoria jurídico-política dos sujeitos haitianos em mobilidade transnacional no Brasil. En R. Baeninger, R. Peres, \& D.

Fernandes (eds.), Imigração haitiana no Brasil. São Paulo, Brasil: Paco Editorial/UNICAMP.

Da Silva, S. (2017). A imigração haitiana e os paradoxos do visto humanitário. En R. Baeninger, R. Peres, \&

D. Fernandes (eds.).Imigração haitiana no Brasil.São Paulo, Brasil: Paco Editorial/UNICAMP.

De Genova, N. (2013). Spectacles of migrant «illegality»: the scene of exclusion, the obscene of inclusion. Ethnic and Racial Studies. doi: 10.1080/01419870.2013.783710

De Genova, N. (2015). Extremities and regularities. Regulatory regimes and the spectacle of immigration enforcement. En Y. Jansen, R. Celikates, \& J. De Bloois (Eds.), The irregularization of migration in contemporary Europe. Detention, deportation, drowning. Maryland, United States: Rowman \& Littlefield International.

De Genova, N. (2018). Rebordering “the People” Notes on Theorizing Populism. South Atlantic Quarterly, 117(2), 357-374.

De Lucas, J. (2003). Inmigración y globalización. Acerca de los presupuestos de una política de inmigración. Redur, 1

Dilla, H. (2016). Chile y sus fronteras: notas para una agenda de investigación. Polis, Revista Latinoamericana, 15(44), 309-327. doi:

Domenech, E. (2009). La visión estatal sobre las migraciones en la Argentina reciente. De la retórica de la exclusión a la retórica de la inclusión. En E. Domenech (ed.), Migración y política: el Estado interrogado. Procesos actuales en Argentina y Sudamérica. Córdoba, Argentina: Universidad Nacional de Córdoba.

Domenech, E. (2013). Las migraciones son como el agua. Hacia la instauración de políticas de control con rostro humano. La gobernabilidad migratoria en la Argentina. Polis, Revista Latinoamericana, 12(35), 119-142.doi: 10.4067/S0718-65682013000200006 
Domenech, E., y Pereira, A. (2017). Estudios migratorios e investigación académica sobre las políticas de migraciones internacionales en Argentina. Iconos, Revista de Ciencias Sociales, (58), 83-108.

Doña, C., \& Mullan, B. (2014). Migration Policy and Development in Chile. International Migration, 52(5), 1-14.

Dufraix, R., y Quinteros, D. (2017). Expulsiones judiciales, sanciones administrativas y derechos fundamentales en la región de Tarapacá, Chile. Presentado en II Jornadas de Migraciones. 26 y 27 de abril, Buenos Aires, Argentina.

Durán, C., y Thayer, L. E. (2017). Los migrantes frente a la Ley: Continuidades y rupturas en la legislación migratoria del Estado Chileno (18241975). Historia 396, 7(2), 429-461.

Feldman-Bianco, B., Cavalcanti, L., \& Araujo, D. (2018). Imigração Haitiana no Brasil. Périplos. Revista de Pesquisa sobre Migrações, 1(1), 1-4.

Fernandes, D., \& de Faria, A. (2017). A diáspora haitiana no Brasil: processo de entrada, característica e perfil. En R. Baeninger, R. Peres, \& D. Fernandes (Eds.), Imigração haitiana no BrasilSão Paulo, Brasil: Paco Editorial/UNICAMP

Freier, F. (2018, julio 18). A South American Migration Crisis: Venezuelan outflows test neighbors' hospitality. Migration Policy Institute.

Freitez, A. (2011). La emigración desde Venezuela durante la última década. Temas de Coyuntura, 63, 11-38.

Garduño, E. (2003). Antropología de la frontera, la migración y los procesos transnacionales. Frontera Norte, 15(30), 1-30.

Ghosh, B. (2008). Derechos humanos y migración: el eslabón perdido. Migración y Desarrollo, 10, 37-63.

González, H. (1998). Características de la inserción de aymaras chilenos y bolivianos en el área de Arica. Taller de Estudios Andinos Arica. Serie documentos de trabajo.

González, I. B. (2010). El control selectivo de las fronteras y la transnacionalización de sus resistencias. Arbor, 186(744), 689-703.

González, S. (2009). Cuadernos Interculturales, 7(13), 27-42.

Grimson, A. (2004). Fronteras, naciones y región. Presentado en Foro Social de las Amércas. Agenda pos-neoliberal, Quito. Ecuador.

Grimson, A. (2005). Fronteras, Estados e identificaciones en el cono sur. En 
D. Mato (ed.), Cultura, Política y Sociedad. Perspectivas Latinoamericanas (pp. 89-101). Buenos Aires, Argentina: CLACSO.

Grimson, A. (2011). Los límites de la cultura. Crítica de las teorías de la identidad. Argentina: Siglo XXI.

Guizardi, M. (2016). El (des) control del Yo. Frontera y simultaneidad en una etnografía sobre las migrantes peruanas en Arica (Chile). Estudios Atacameños, 53.

Guizardi, M., y Garcés, A. (2012). Mujeres peruanas en las regiones del norte de Chile. Apuntes preliminares para la investigación. Estudios Atacameños, 44, 5-34.

Guizardi, M. L., Valdebenito, F., López, E., y Nazal, E. (2015). Condensaciones en el espacio hiperfronterizo: Apropiaciones migrantes en la frontera norte de Chile. En M. L. Guizardi (Ed.), Las Fronteras del Transnacionalismo. Límites y desbordes de la experiencia migrante en el centro y norte de Chile. Santiago, Chile: Universidad de Tarapacá y Ocho Libros.

Harvey, D. (2003). The Condition of Posmodernity. An enquiry into the origins of cultural change. Oxford, UK: Blackwell.

International Organization for Migration OIM. (2018, febrero). National Migration Trends in South America. Bolivarian Republic of Venezuela.

Karakayali, S. (2015). Illegal migration in Post Fordism. En Y. Jansen, R. Celikates, \& J. De Bloois, The irregularization of migration in contemporary Europe. Definition, Deportation, Drowning. London, UK: Rowman \& Littlefield International.

Kearney, M. (1991). Borders and Boundaries of State and Self at the End of Empire. Journal of Historical Sociology, 4(1), 52-74.

Kearney M. (1995). The Local and the Global: The anthropology of globalization and transnationalism. Annual Review of Anthropology, 24, 547-565.

Kearney, M. (2004). The classifying and value-filtering missions of borders. Anthropological Theory, 4(2), 131-156.

Kolossov, V. (2005). Border Studies: Changing perspectives and theoretical approaches. Geopolitics, 10(4), 606-632.

Kretsedemas, P., \& Brotherton, D. (eds.). (2018). Immigration Policy in the Age of Punishment: Detention, Deportation, and Border Control. New York, United States: Columbia University Press. 
Lara, M. D., y Santos, T. (133d. C.). La detención policial de las personas extranjeras en las regiones del norte de Chile. Nova Criminis, 8(12), 2016.

Lefebvre, H., \& Levich, C. (1987). The Everyday and Everydayness. Yale French Studies, 73, 7-11.

Levitt P., \& Glick Schiller N. (2004). Conceptualizing simultaneity: A transnational social field perspective on society. International Migration Review, 38(3), 1002-1039.

Mamed, L., \& Oliveira de Lima, E. (2017). Movimento de trabalhadores haitianos para o Brasil nos últimos cinco anos: a rota de acesso pela Amazonia Sul Ocidental e o acampamento público de imigrantes no Acre. En R. Baeninger, R. Peres, \& D. Fernandes (Eds.), Imigração haitiana no Brasil. São Paulo, Brasil: Paco Editorial/UNICAMP.

Mármora, L. (2010). Modelos de gobernabilidad migratoria. La perspectiva política en América del Sur. Revista Interdisciplinar da Mobilidade Humana - REMHU, 18(35).

Martínez, J., y Orrego, C. (2016). Nuevas tendencias y dinámicas migratorias en América Latina y el Caribe (Población y Desarrollo No. 114). Santiago, Chile: CEPAL, OIM.

Mezzadra, S. (2016). What's at stake in the mobility of labour? Borders, migration, contemporary capitalism. Migration, Mobility and Discplacement, 2(1), 30-43.

Mezzadra, S., \& Neilson, B. (2012). Between Inclusion and Exclusion: On the Topology of Global Space and Borders. Theory, Culture and Society, 29(4), 58-75.

Mezzadra, S., \& Neilson, B. (2013). Border as method or the multiplication of labor. Durham, United States: Duke University Press.

Modolo, V. (2010). La movilidad territorial en el Mercado Común Europeo y Mercosureño. En S. Novick, G. Mera, y V. Modolo (Eds.), Migraciones y Mercosur: una relación inconclusa. Buenos Aires, Argentina: Catálogos.

Mora, C., y Undurraga, E. (2013). Racialisation of inmigrants at work: Labour mobility and segmentation of peruvian migrants in Chile. Bulletin of Latin American Research, 32(3), 294-310.

Munck, R. (2012). Migration, development and work in the global order: a new perspective. Journal of the Office of Latino/Latin-American Studies, 4(1), 42-56. 
Nestor García Canclini. (1990). Culturas Hibridas. Estrategias para entrar y salir de la modernidad. Ciudad de México, México: Grijalbo.

Norambuena, C. (2013). Amenazas sin fronteras: nuevas periferias en la circulación de personas. Cuadernos de Historia, 39, 147-168.

Norambuena Carrasco, C. (1990). Política y legislación inmigratoria en Chile, 1830-1930 (Vol. 10). Santiago, Chile: Universidad de Chile.

Novick, S., Mera, G., y Modolo, V. (eds.). (2010). Migraciones y Mercosur: una relación inconclusa. Buenos Aires, Argentina: Catálogos.

OEA CIDH. (2015). Movilidad Humana, Estándares interamericanos. Derechos Humanos de migrantes refugiados, apátridas, víctimas de trata de personas y desplazados internos: normas y estándares del sistema Interamericano de Derechos Humanos (OEA/Ser.L/V/II. No. 46/15). CIDH OEA.

OIM, IPPDH, \& MERCOSUR. (2017). Diagnóstico regional sobre migración haitiana. Buenos Aires, Argentina: OIM.

Piper, N. (2017). Global governance of labour migration: From 'management' of migration to an integrated rights-based approach. En P. Drahos (Ed.), Regulatory Theory. Foundations and applications. ANU Press.

Portes, A., Guarnizo, L., \& Landolt, P. (2003). El estudio del transnacionalismo: Peligros latentes y promesas de un campo de investigación emergente. En A. Portes, L. Guarnizo, \& P. Landolt (eds.), La globalización desde abajo: transnacionalismo inmigrante y desarrollo. La experiencia de Estados Unidos y América Latina (p. 397). Ciudad de México: México: Miguel Angel Porrúa; FLACSO México.

Prescott, J. R. V. (1978). TBoundaries and Frontiers. Londres, UK: Croom Helm.

Quinteros, D. (2016). ¿ ¿Nueva «crimigración» o la vieja economía política del castigo? Dos aproximaciones criminológicas para entender el control punitivo de la migración en Chile. Revista Astrolabio. Nueva Eроса, 17, 81-113.

Rojas, N., y Silva, C. (2016). La migración en Chile: Breve reporte y caracterización. Observatorio Iberoamericano sobre Movilidad Humana. Migración y Desarrollo, OBIMID.

Rojas, N., Silva, C., Amode, N., Vásquez, J., y Orrego, C. (2017). Migración haitiana en Chile (Boletín informativo No. 1). Santiago, Chile: Departamento de Extranjería y Migración. 
Rumford, C. (2006). Theorizing Borders. European Journal of Social Theory, 9(2), 155-169.

Salazar Parrenas, R. (2000). Migrant Filipina Domestic Workers and International Division of Reproductive Labor. Gender and Society, 14(4), 560-580.

Salter, M. (2016). The global visa regime and the political technologies of the international self: Borders, bodies, biopolitics. Alternatives, 31, 167-189.

Sanahuja, J. A. (2017). Posglobalización y ascenso de la extrema derecha: crisis de hegemonía y riesgos sistémicos. En M. Mesa (Ed.), Seguridad internacional y democracia: guerras, militarización y fronteras. Anuario 2016-2017 (pp. 41-78). Madrid, España: Fundación Cultura de Paz.

Sausi, J. L., y Oddone, N. (2010). Cooperación e integración transfronteriza en el Mercosur: el caso de la Triple Frontera Argentina-Brasil-Paraguay. En L. Maira (Ed.), La política internacional subnacional en América Latina (pp. 209-258). Buenos Aires, Argentina: DelZorzal.

Sørensen, N., \& Gammeltoft Hansen, T. (2013). Introduction. En N. Sørensen \& T. Gammeltoft Hansen (Eds.), The Migration Industry and the Commercialization of International Migration (Global Institutions) (pp. 1-23). Nueva York, United States: Routledge.

Stefoni, C. (2011). Ley y política migratoria en Chile. La ambivalencia en la comprensión del migrante. En B. Feldman-Bianco, L. Rivera Sánchez, C. Stefoni, \& M. I. Villa (Eds.), La construcción social del sujeto migrante en América Latina: prácticas, representaciones y categorías (pp. 79-109). Quito, Ecuador: FLACSO-Ecuador/ Santiago, Chile: CLACSO, Universidad Alberto Hurtado.

Stefoni, C. (2018a). Panorama de la migración internacional en América del Sur. Documento elaborado en el marco de la Reunión Regional Latinoamericana y Caribeña de Expertas y expertos en Migración Internacional preparatoria del Pacto Mundial para una Migración Segura, Ordenada y Regular. (No. Serie Población y Desarrollo 123). CEPAL/OIM.

Stefoni, C. (2018b, junio 29). Chile ha sido el país menos solidario con la crisis humanitaria que atraviesa Haití. El desconcierto www.eldesconcierto.cl

Stefoni C., Silva C. (2018) Migración Venezolana en Chile en: Koechlin, José, y Joaquín Eguren, eds. El éxodo venezolano: entre el exilio y la emigración. Lima, Perú: Konrad Adenauer Stiftung, Antonio Ruiz de Montoya, OIM, OBIMID, 2018. 
Sum, N. L. (2003). Rethinking Globalisation: Re-articulating the Spatial Scale and Temporal Horizons of Trans-Border Spaces. En N. Brenner, B. Jessop, M. Jones, \& G. Macleod (Eds.), State/Space: A Reader.(pp. 208-224). Oxforf: Blackwell Publishing.

Tapia, M., y González, A. (2014). Fronteras, Regiones Fronterizas y Migraciones. Entre apertura, integración y cierre. En M. Tapia y A. González (eds.), Regiones transfronterizas. Migración y los desafíos para los estado nacionales latinoamericanos.Santiago, Chile:RIL Editores.

Tapia, M., y Parella, S. (2015). Las regiones fronterizas para el estudio de la migración y la circulación. Un análisis a partir de dos casos ilustrativos. En M. Guizardi (Ed.), Las fronteras del transnacionalismo. Límites y desbordes de la experiencia migrante en el centro y norte de Chile. Santiago. Chile: Ocholibros. Universidad de Tarapacá.

Texidó, E., y Gurrieri. (2012). Panorama migratorio de América del Sur. OIM (Perfiles Migratorios). Buenos Aires, Argentina: OIM.

Thayer, E. (2015). Territorio, democracia en crisis y migración transnacional: el estado chileno frente a la nueva pluralidad social. En M. Guizardi (Ed.), Las fronteras del transnacionalismo. Límites y desbordes de la experiencia migrante en el centro y norte de Chile. Santiago. Chile: Ocholibros. Universidad de Tarapacá.

Thayer, E. (2016). Migración, Estado y seguridad. Tensiones no resueltas y paradojas persistentes. Polis, Revista Latinoamericana15(44), 109129. doi:10.4067/S0718-65682016000200006

UNHCR The UN Refugee Agency. (2018). Venezuela Situation. Responding to the needs of people displaced from Venezuela. Supplementary appeal.

Vallet, E. (2014). Borders, Fences and walls. State of insecurity?London, UK: Routledge.

Vásquez, T., Busse, E., \& Izaguirre, L. (2015). Migración de población haitiana a Perú y su tránsito hacia Brasil desde elaño 2010. Lima, Perú: Instituto de Estudios Peruanos IEP.

Velasco, L., y Contreras, O. (2011). Introducción. En Mexican Voices of the border region. Philadelphia, United States: Temple University Press.

Vila, P. (2000). Crossing Borders, Reinforcing Borders. Austin, United States: University of Texas Press.

Wallerstein, I. (1995). La estructura interestatal del sistema-mundo moder- 
no. Secuencia, Revista de Historia y Ciencias Sociales, 32, nueva época, 143-166.

Wimmer, A., \& Glick Schiller, N. (2003). Methodological nationalism, the social sciences, and the study of migration: An essay in historical epistemology. International Migration Review, 37(3), 576-610.

Zapata-Barrero, R. (2013). Fundamentos de las políticas interculturales en las ciudades: Respuestas a tres preguntas frecuentes. En Manual para el diseño de políticas interculturales (pp. 45-63). Barcelona, España: Universitat Pompeu Fabra. 